\title{
Reflection Asymmetry in Ra Isotopes
}

\author{
E.V. Mardyban ${ }^{1,2}$, T.M. Shneidman ${ }^{1,3}$, N.V. Antonenko ${ }^{1}$, \\ G.G. Adamian ${ }^{1}$ \\ ${ }^{1}$ Bogoliubov Laboratory of Theoretical Physics, \\ Joint Institute for Nuclear Research, Dubna 141980, Russia \\ ${ }^{2}$ Dubna State University, 141982 Dubna, Moscow Region, Russia \\ ${ }^{3}$ Kazan Federal University, 420008, Kazan, Russia
}

Received 16 December 2021

doi: https://doi.org/10.55318/bgjp.2022.49.1.078

\begin{abstract}
We developed a model which allows to take into account both shape deformation parameters and cluster degrees of freedom. The important ingredient of the model is the dinuclear system concept in which the wave function of the nucleus is treated as a superposition of a mononucleus and two-cluster configurations. The model is applied to describe the properties of alternating rotational bands in $222,224,226,228 \mathrm{Ra}$. The spin-dependences of the parity splitting and of the reduced matrix elements of $E 1$ transitions between the members of alternating parity bands are calculated and compared with experimental data.
\end{abstract}

KEY WORDS: ENTER PLEASE KEY WORDS OR PHRASES IN ALPHABETICAL ORDER, SEPARATED BY COMMAS.

\section{Introduction}

In the even-even isotopes of actinides the low-lying negative parity states with energies lower than the two-quasiparticle limit are observed. The lowest negative-parity states form the rotational or quasivibrational bands which mainly decay via collective $E 1$ transitions into the ground-state band. While the formation of the positive-parity bands is connected in general to the quadrupole collective motion, the lowering of states with negative-parity is a signature of the reflection asymmetric collective mode. From the microscopic point of view, the possibility of strong reflection-asymmetric correlations in the vicinity of the ground state can be associated with the appearance of orbital pairs with $\Delta j=\Delta l=3$ near the Fermi surface. Besides the actinides, a similar situation occurs in nuclei with masses near $A \sim 56$ and $A \sim 134$ that is in agreement with experimental data. The results of calculations within the shell-corrected liquid drop models [1,2] and mean-field models [3-5] show that nuclei in these 


\section{Reflection Asymmetry in Ra Isotopes}

mass regions are either soft with respect to the octupole deformation or octupoledeformed. The possibility of the strong octupole correlations in actinides was also investigated in the frame of the coherent quadrupole-octupole collective model [6].

An alternative explanation is suggested by the dinuclear system model in which long-range multipole correlations might lead to the formation of light clusters on the surface of a heavy nucleus. The contribution of such dinuclear configurations to the nuclear wave function naturally leads to an appearance of a reflection-asymmetric deformation. The strength of this deformation is determined by the relative weight of the dinuclear configurations. The idea that clustering is responsible for the reflection-asymmetric deformations of the medium mass and heavy nuclei is based on the fact that these nuclei are good alphaemitters. Moreover, the calculation of potential energies of the dinuclear systems with $\alpha$-particle as a light cluster have shown that their energies are close to the binding energy of the corresponding nucleus. Thus, there is a significant probability to form an $\alpha$-cluster in the surface region of the nucleus.

In Ref. [7,8], the dinuclear model have been used to describe the parity splitting in actinides and rare-earth nuclei. To describe the reflection asymmetric collective modes characterized by nonzero values of angular momentum projection $K$, the degrees of freedom related to the internal excitation of clusters were taken into account $[9,10]$. The excited $0^{+}$states in ${ }^{240} \mathrm{Pu}$ which decays via strong $E 1$ transition to the members of lowest negative-parity band was explained as a first excited state in mass asymmetry degree of freedom [11]. Recently, the alphacluster model have been successfully applied to describe recent experimental data on the strength of $B(E 2)$ transitions in the ground-state band of ${ }^{212} \mathrm{Po}$ [12].

In a series of recent experiments $[13,14]$ conducted at HIE-ISOLDE facility at CERN, an electric dipole and octupole transitional moments have been measured in ${ }^{222,224,228} \mathrm{Ra}$. It is interesting that despite the fact that the parity splitting has a minimum at ${ }^{224} \mathrm{Ra}$, the dipole and octupole moments continue to increase in ${ }^{222} \mathrm{Ra}$ providing evidence for increasing reflection-asymmetric deformation as expected from the dinuclear system model [8]. The weight of alpha-cluster dinuclear system is expected to increase all the way up to ${ }^{220} \mathrm{Ra}$ thus increasing the octupole and dipole transition probabilities. The increase of parity splitting for light $\mathrm{Ra}$ isotopes can be understood as due to the interplay between quadrupole deformation of the heavy cluster and mass-asymmetry degree of freedom.

In this work we apply the dinuclear system model to analyze the isotopic dependence of parity splitting and of the electric dipole transition probabilities in $\mathrm{Ra}$ isotopes. 
E.V. Mardyban, T.M. Shneidmanr, N.V. Antonenko, G.G. Adamian

\section{Model}

\subsection{Hamiltonian}

Instead of parametrizing nuclear shape in terms of multipole deformation parameters, we use degrees of freedom related to the dinuclear system (DNS). As DNS we understand the system of two touching fragments $\left(A_{1}, Z_{1}\right)$ and $\left(A_{2}, Z_{2}\right)$ with $A_{1}+A_{2}=A$ and $Z_{1}+Z_{2}=Z$ kept together by the molecular-type nucleus-nucleus potential. The special case of the DNS in which one fragment has zero mass is denoted as a mononucleus. Each dinuclear system configuration can be characterized by the mass-asymmetry $\xi=2 A_{2} / A$, charge-asymmetry $\xi_{Z}=2 Z_{1} / Z$, and the vector of relative distance between the centers of the fragments $\mathbf{R}=\left(R, \theta_{R}, \phi_{R}\right)$.

The main idea of the DNS model is that the intrinsic nuclear wave function can be assumed as a superposition of the monunucleus and different dinuclear configurations. The mononucleus is taken to be quadrupole-deformed; thus, in the DNS model, the reflection-asymmetric deformation of the nucleus is related solely to the nonzero weight of the asymmetric dinuclear systems.

Applying the model to the description of the low-lying excitations in actinides, we presume that the main source of the reflection asymmetric deformation is the contribution of the DNS with an alpha-particle as a light cluster. As was mentioned in the Introduction, actinides are good $\alpha$-emitters, thus, there is a significant probability to form an $\alpha$-cluster in the surface region of the nucleus. Due to the stable closed-shell structure of the alpha-cluster, it is reasonable to presume that the ground-state and low-lying excited states of actinides can have a significant contribution of $\alpha+(A-4, Z-2)$ cluster structures $(\alpha$-cluster DNS) without appreciable internal excitations of the heavy fragment.

The degrees of freedom chosen to characterize a system with quadrupole deformed heavy cluster and spherical light cluster are related to the transfer of nucleons between the fragments, to the rotation of the heavy fragment, and to the relative motion of fragments. The charge asymmetry $\xi_{Z}$ is not considered as an independent collective variable and fixed to ensure neutron-to-proton ratio in the light fragment to be as in alpha-particle, i.e. $Z_{2} / A_{2}=0.5$.

The Hamiltonian of the model has the following form

$$
\begin{aligned}
\hat{H}_{0}=-\frac{\hbar^{2}}{2 B_{\xi}} \frac{1}{\xi^{3 / 2}} \frac{\partial}{\partial \xi} \xi^{3 / 2} & \frac{\partial}{\partial \xi}+\frac{\hbar^{2}}{2 \Im_{h}(\xi)} \hat{l}_{h}^{2} \\
& +\frac{\hbar^{2}}{2 \mu(\xi) R_{m}^{2}} \hat{l}_{0}^{2}+\frac{\hbar \omega_{R}(\xi)}{2}+U\left(\xi, R_{m}, \beta_{0}, \epsilon\right) .
\end{aligned}
$$

Here, we assumed that the value of internuclear distance $R=R_{m}\left(\xi, \beta_{0}, \epsilon\right)$ is set equal to the position of the minimum of the potential in $R$ for given value of $\xi$, relative orientation of the fragments defined by angle $\epsilon$ and the deformation 


\section{Reflection Asymmetry in Ra Isotopes}

$\beta_{0}$ of the heavy fragment. The heavy fragment is assumed to be spherical or quadrupole deformed. The calculations of the potential energy [15] show that the minimum corresponds to the touching configuration of the clusters. The energy of the zero-order vibrations in $R$ around the equilibrium value $R_{m}$ is given as $\hbar \omega_{R} / 2$. The mass-asymmetry is treated as a continuous variable. In (1), $\mu \approx m_{0} A \xi / 2$ is the reduced mass of the DNS $(\xi \ll 1), m_{0}$ is the nucleon mass, and $\Im_{h}(\xi)$ is the moment of inertia of the heavy fragment. The angular momentum operators describing the rotation of the heavy fragment, $\hat{l}_{h}^{2}$, and the relative rotation, $\hat{l}_{0}^{2}$, are given as

$$
\stackrel{2}{i}=-\frac{1}{\sin \theta_{i}} \frac{\partial}{\partial \theta_{i}} \sin \theta_{i} \frac{\partial}{\partial \theta_{i}}-\frac{1}{\sin ^{2} \theta_{i}^{2}} \frac{\partial^{2}}{\partial \phi^{2}}, \quad(i=0, h) .
$$

The angles $\Omega_{i}=\left(\theta_{i}, \phi_{i}\right)$ describe the orientation of the symmetry axis of the heavy fragment $(i=h)$ and of the vector $\mathbf{R}(i=0)$ with respect to the laboratory frame. The angle of the relative orientation $\epsilon$ cane be related to $\Omega_{0}$ and $\Omega_{h}$ by the expression

$$
\sin ^{2} \epsilon=\frac{2}{3}\left(1-\sqrt{5}\left[Y_{2}\left(\Omega_{h}\right) \times Y_{2}\left(\Omega_{0}\right)\right]_{00}\right) .
$$

The calculation of the mass parameter $B_{\xi}$ is given in Ref. [16]. In the current work $B_{\xi}$ is treated as a parameter of the model.

The potential energy $U\left(\xi, R_{m}, \beta_{0}, \epsilon\right)$ is determined as

$$
U\left(\xi, R_{m}, \beta_{0}, \epsilon\right)=B_{1}(\xi)+B_{2}(\xi)+V\left(\xi, R_{m}, \beta_{0}, \epsilon\right),
$$

where the quantities $B_{1}$ and $B_{2}$ are the binding energies of the clusters forming the DNS. The experimental ground-state masses from Ref. [17], if available, are used in the calculations. If not, the predictions of Ref. [2] are used. Shell effects and pairing correlations are included in the binding energies. The nucleusnucleus potential $V\left(\xi, R_{m}, \beta_{0}, \epsilon\right)$ is calculated using the procedure described in Ref. [15].

The potential energy as a function of relative orientation of the fragments has a minimum which corresponds to the pole-to-pole orientation $(\epsilon=0)$. We approximate the $\epsilon$-dependence of the potential energy by the second order expansion in the Legendre polynomials as

$$
U\left(\xi, R_{m}, \beta_{0}, \epsilon\right)=U_{0}\left(\xi, R_{m}, \beta_{0}\right)+C_{0} \beta_{0} \xi \sin ^{2} \epsilon,
$$

where $U\left(\xi, R_{m}, \beta_{0}, \epsilon\right)=U_{0}\left(\xi, R_{m}, \beta_{0}\right)=U\left(\xi, R_{m}, \beta_{0}, \epsilon=0\right)$. The second term in Eq. (5) describes the interaction between the rotational degrees of freedom and mass asymmetry. Since in the vicinity of the ground state both $\xi \ll 1$ and $\beta_{0} \ll 1$, we expand the interaction in Eq. (5) leaving only term which is linear in $\xi$ and $\beta_{0}$. 


\subsection{Diagonalization of the Hamiltonian}

The Hamiltonian (1) is diagonalized on the set of basis functions

$$
\Phi_{L, M, \pi}^{l_{1}, l_{2}, n}=F_{n}(\xi)\left[Y_{l_{1}}\left(\Omega_{h}\right) \times Y_{l_{2}}\left(\Omega_{\mathbf{R}}\right)\right]_{(L M)},
$$

where $n=0,1,2 \ldots, l_{1}=0,2,4 \ldots$, and $l_{2}=0,1,2 \ldots$ The basis functions in mass asymmetry are denoted as $F_{n}(\xi)$. The angular part of the basis wave function (6) is given by the bipolar spherical harmonics, which provide the proper transformation properties with respect to the rotation and space inversion. Since we have assumed that the heavy fragment is the axially-symmetric quadrupole rotator the quantum number $l_{1}$ can take only even values. The parity of the state is then determined as $\pi=(-1)^{l_{2}}$.

The peculiarities of the spectra of Hamiltonian (1) strongly depend on the strength of the interaction between the rotational and mass-asymmetry degrees of freedom. To qualitatively analyze the role of this interaction, lets assume that the mass-asymmetry motion is significantly faster than both the rotational motion of the heavy fragment and the relative rotation. Then in the description of the yrast spectrum we can separate the motion in $\xi$ from the other degrees of freedom and consider the system in its lowest state $\Psi_{0}(I, \xi)$ with respect to the mass-asymmetry [9]. We can then average out the Hamiltonian (1) with respect to $\xi$ and obtain its simplified form as

$$
\hat{H}_{0}=\frac{\hbar^{2}}{2 \Im_{h}\left(\xi_{0}(I)\right)} \hat{l}_{h}^{2}+\frac{\hbar^{2}}{2 \mu\left(\xi_{0}(I)\right) R_{m}^{2}} \hat{l}_{0}^{2}+C_{0} \beta_{0} \xi_{0}(I) \sin ^{2} \epsilon,
$$

where $\xi_{0}(I)=\left\langle\Psi_{0}(I)|\xi| \Psi_{0}(I)\right\rangle$ is the average mass asymmetry for the angular momentum $I$.

If for certain angular momentum either $\beta_{0} \approx 0$ or $\xi_{0} \approx 0$, then the interaction term in Eq. (7) can be approximately neglected. This leads to the yrast spectrum of the form

$$
\begin{array}{ll}
E(I)=\frac{\hbar^{2}}{2 \Im_{h}} I(I+1), & (\text { even } I), \\
E(I)=\frac{\hbar^{2}}{2 \Im_{h}} I(I+1)+\frac{\hbar^{2}}{2 \mu R_{m}^{2}}, & (\text { odd } I) .
\end{array}
$$

Thus, the negative-parity band is just shifted on an amount $\hbar^{2} /\left(2 \mu R_{m}^{2}\right)$ with respect to the positive parity ground state band.

The opposite case of strong interaction occurs when neither $\xi_{0}$ nor $\beta_{0}$ are small. In this case the light fragment is localized in the pole region of the heavy one. As was shown in Appendix of [10], in the limit of strong interaction, i.e. $C_{0} \xi_{0}(I) \beta_{0} \gg 1$, the Hamiltonian can be diagonalized analytically, yielding the spectrum of the form

$$
E(I)=\frac{\hbar^{2}}{2\left(\Im_{h}+\mu R_{m}^{2}\right)} I(I+1), \quad I=0,1,2, \ldots
$$




\section{Reflection Asymmetry in Ra Isotopes}

Such a spectrum is expected for the rotor with stable reflection-asymmetric deformation.

Since the moments of inertia of dinuclear systems is generally larger than that of the mononucleus configuration, the energies of cluster configurations grow slower with angular momentum. Because of this, the weights of cluster components and $\xi_{0}(I)$ are both increasing with $I$. Therefore, we expect that the yrast spectrum of the nucleus will evaluates with spin from the limit of reflectionasymmetric vibrations, Eq. (8), to the limit of unperturbed band with interleaved positive and negative parity states given by Eq. (9).

\subsection{Multipole moments}

The reduced transition probability for the transition from the initial state $\mid i>$ to the final state $\mid f>$ is calculated as

$$
B(E \lambda ; i \rightarrow f)=\frac{1}{2 I_{i}+1}\left|\left\langle f\left\|Q_{\lambda}\right\| i\right\rangle\right|^{2},
$$

where the multipole operator $Q_{\lambda \mu}$ is defined as

$$
Q_{\lambda \mu}=\int \rho_{Z}(\mathbf{r}) r^{\lambda} Y_{\lambda \mu}(\Omega) d \mathbf{r} .
$$

To obtain the expression for the multipole operator in the DNS, we have to substitute the charge density operator $\rho_{Z}(\mathbf{r})$ in (11) by

$$
\rho_{Z}(\mathbf{r})=\rho_{Z_{1}}(\mathbf{r})+\rho_{Z_{2}}(\mathbf{r}),
$$

where $\rho_{Z_{i}}(\mathbf{r})(i=1,2)$ are the charge densities of the DNS fragments. The expressions for the dipole and octupole operators then become

$$
\begin{aligned}
Q_{1 \mu}= & \epsilon_{1} \sqrt{4 \pi} \frac{A_{1} Z_{2}-A_{2} Z_{1}}{A} R\left\{Y_{0}\left(\Omega_{h}\right) \times Y_{1}\left(\Omega_{R}\right)\right\}_{1 \mu} \\
Q_{3 \mu}= & \epsilon_{3} \sqrt{4 \pi} \frac{A_{1}^{3} Z_{2}-A_{2}^{3} Z_{1}}{A^{3}} R^{3}\left\{Y_{0}\left(\Omega_{h}\right) \times Y_{3}\left(\Omega_{R}\right)\right\}_{3 \mu} \\
& -4 \pi \sqrt{\frac{7}{5}} Q_{0} \frac{A_{2}}{A} R\left\{Y_{2}\left(\Omega_{h}\right) \times Y_{1}\left(\Omega_{R}\right)\right\}_{3 \mu},
\end{aligned}
$$

where $\epsilon_{1}$ and $\epsilon_{3}$ are the effective charges introduced for dipole and octupole transitions, respectively. As follows from Eq. (13), the dipole operator has only contribution from the DNS configurations, while the octupole operator has a nonzero contribution from the mononucleus configuration as well.

\section{Results of Calculations}

The yrast energy spectra of ${ }^{222,224,226,228} \mathrm{Ra}$, calculated by diagonalizing the Hamiltonian (1), are presented in Figure 1. The effective mass parameter for the 


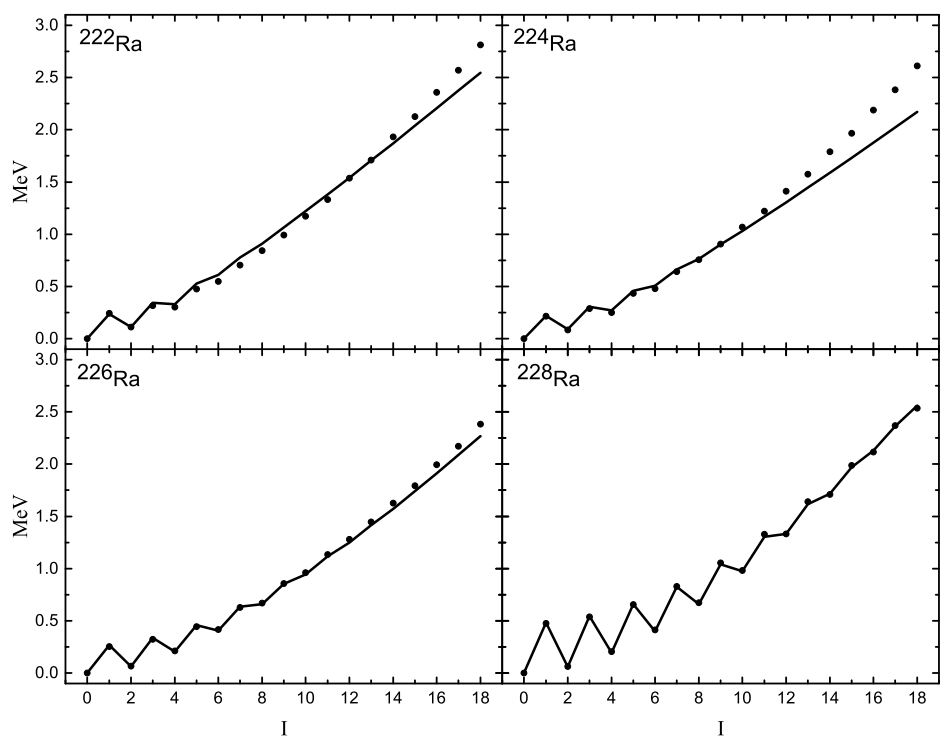

Figure 1. Comparison of experimental (points) and calculated (solid lines) energies of states of the alternating parity bands in ${ }^{222,224,226,228} \mathrm{Ra}$. Experimental data are taken from Ref. [20]. Calculated energies are obtained by the numerical diagonalization of the Hamiltonian (1).

mass asymmetry motion is fixed as $B_{\xi}=10 \times 10^{5} \mathrm{~m}_{0} \mathrm{fm}^{2}$ for all considered nuclei. One should note that this value is larger than those used in Ref. [8] because of the fact that additional degrees of freedom are considered. The potential energy is taken in the form of Eq. (5). The value of $C_{0}$ is fixed by approximating the $\epsilon$-dependence of the potential energy calculated with Eq. (4). The potential energy of the mononucleus configuration $U_{0}\left(\xi=0, \beta_{0}\right)$ and the moment of inertia of the heavy fragment $\Im_{h}(\xi)$ can not be calculated within the DNS model. Instead, $U_{0}$ is fitted to reproduce the correct value of the nuclear binding energy as the lowest eigenstate of the Hamiltonian (1). The moment of inertia is parameterized in the form

$$
\Im_{h}(\xi)=c(\xi) \Im_{h}^{(r i g)}(\xi),
$$

where $\Im_{h}^{(r i g)}(\xi)$ is the rigid body moment of inertia for the corresponding heavy fragment and

$$
c(\xi)= \begin{cases}0.85, & \xi>\xi_{\alpha}, \\ c_{0}+\left(0.85-c_{0}\right) \xi / \xi_{\alpha}, & \xi \leq \xi_{\alpha} .\end{cases}
$$

The quantity $c_{0}$ is a scaling parameter which is fixed by the calculation of the energy of the $2^{+}$state of the ground-state band. Calculations show that for considered nuclei $c_{0}=(0.2-0.45)$. 


\section{Reflection Asymmetry in Ra Isotopes}

As seen, the agreement between the calculated spectra and the corresponding experimental rotational bands are rather good for all considered $\mathrm{Ra}$ isotopes. One can see that the structure of the spectra is similar for all nuclei. In the beginning of the band, the negative parity states are shifted up with respect to the positive parity ones. As expected, because the weight of cluster components increasing with angular momentum, the shift between negative and positive parity states decreases. At certain critical value of angular momentum, the smooth rotational band with interleaved positive and negative parity states is formed. This angular momentum corresponds to the transition to the limit of stable reflectionasymmetric deformation [18].

The transition from the limit of octupole vibration to the rotation of stable octupole-deformed nucleus can be conveniently analyzed using the parity splitting quantity defined in Ref. [19]

$$
S\left(I^{-}\right)=E\left(I^{-}\right)-\frac{(I+1) E_{(I-1)}^{+}+I E_{(I+1)}^{+}}{2 I+1},
$$

which provides zero value for the rotational band of nucleus with rigid octupole deformation. Having maximum value in the beginning of the band, the parity splitting decreases with increasing angular momentum. The calculated and experimental dependences of the parity splitting on $I$ are presented in Figure 2. In

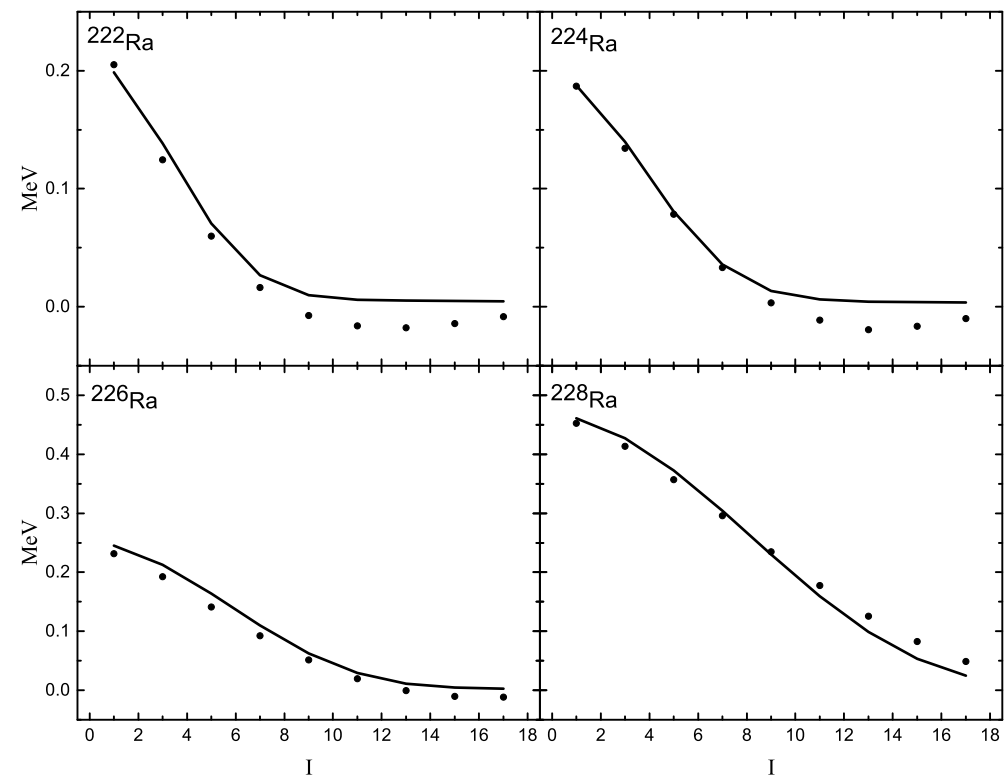

Figure 2. Caculated (lines) and experimental (points) values of parity splitting (see Eq. (16)) for ${ }^{222,224,226,228} \mathrm{Ra}$. Experimental energies are taken from [20]. 
our model the value of parity splitting is determined by the relative weights of the cluster components and the mononucleus in the wave function. Due to the larger moment of inertia the weight of $\alpha$-particle DNS increases with angular momentum that decreases the parity splitting.

Using the wave functions obtained by the diagonalization of the Hamiltonian (1) and the dipole operator given in Eq. (13), we calculated the reduced matrix elements for the dipole transitions between the positive and negative parity states in Ra isotopes. Results are presented in Figure 3. For the ${ }^{222,228} \mathrm{Ra}$ and ${ }^{226} \mathrm{Ra}$ the experimental data are taken from Ref. [14] and Ref. [21], respectively. For ${ }^{224} \mathrm{Ra}$ the experimental data are from Ref. [13]. The effective charge for $E 1$ transitions has been taken to be equal to $\epsilon_{1}=e(1+\chi)$ with an average state-independent value of the $E 1$ polarizability coefficient $\chi=-0.7$ [22]. This renormalization takes into account a coupling of the mass-asymmetry mode to the giant dipole resonance in a dinuclear system. The calculations qualitatively reproduce the angular momentum dependence of the experimental matrix elements in ${ }^{222,224,228} \mathrm{Ra}$. We see again that increase of weights of the cluster systems leads to the enhancement of the strength of $E 1$ transitions.

Note that the model has failed to reproduce anomalously small magnitude of $E 1$ transition strength in ${ }^{224} \mathrm{Ra}$. The possible explanation is following. As shown in Ref. [23], the strength of dipole transitions is very sensitive to the proton-neutron

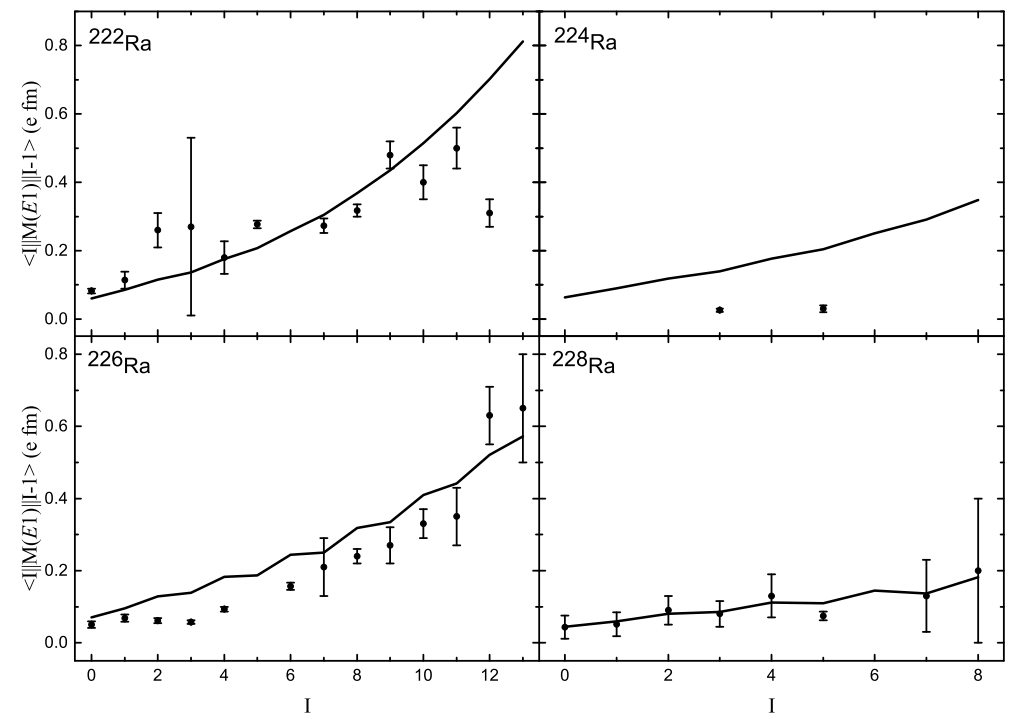

Figure 3. Angular momentum dependencies of the calculated reduced matrix elements of the electric dipole operator (solid lines) between the states of alternating parity bands in ${ }^{222,224,226,228} \mathrm{Ra}$. Experimental data (points) are taken from Ref. [14] for ${ }^{222,228} \mathrm{Ra}$, from Ref. [13] for ${ }^{224} \mathrm{Ra}$, and from Ref. [21] for ${ }^{226} \mathrm{Ra}$. 


\section{Reflection Asymmetry in Ra Isotopes}

ratios in the clusters. Therefore, the independent treatment of collective motion in mass and charge asymmetry is required to describe accurately the peculiarities of dipole transition strength along the isotopic chain and, in particular, the strong decrease of $B(E 1)$ in ${ }^{224} \mathrm{Ra}$. This work is currently in progress.

\section{Conclusion}

We have suggested a cluster interpretation of the properties of the alternating parity bands in heavy nuclei assuming collective motion in the mass asymmetry degree of freedom and by taking into account the relative angular motion of clusters. It was demonstrated that the transition from the limit of reflectionasymmetric shape vibrations to the stable reflection-asymmetric deformation, which occurs with increase of angular momentum, can be associated with increasing weights of cluster components in the nuclear wave function. The existing experimental data on the angular momentum dependence of parity splitting and of reduced matrix elements of dipole transitions between the members of alternating parity bands are well reproduced for ${ }^{222,226,228} \mathrm{Ra}$ isotopes. The strength of dipole transitions is overestimated for ${ }^{224} \mathrm{Ra}$. Independent treatment of mass and charge asymmetry degrees of freedom is required in order to describe anomalously small $B(E 1)$ values in ${ }^{224} \mathrm{Ra}$.

\section{Acknowledgments}

This work was partly supported by the RFBR (Grant No. 20-02-00176). T.M.S. acknowledges the support from the Russian Government Subsidy Program of the Competitive Growth of Kazan Federal University.

\section{References}

[1] W. Nazarewicz et al. (1984) Nucl. Phys. A 429269.

[2] P. Möller et al. (2008) At. Data Nucl. Data Tables 94758.

[3] J. Egido, L. Robledo (1989) Nucl. Phys. A 49485.

[4] K. Rutz, J.A. Maruhn, P.-G. Reinhard, W. Greiner (1995) Nucl. Phys. A 590680.

[5] L.M. Robledo, G.F. Bertsch (2011) Phys. Rev. C 84054302.

[6] N. Minkov, S. Drenska, M. Strecker, W. Scheid, H. Lenske (2012) Phys. Rev. C 85 034306.

[7] T.M. Shneidman, G.G. Adamian, N.V. Antonenko, R.V. Jolos, W. Scheid (2002) Phys. Lett. B $\mathbf{5 2 6} 322-328$.

[8] T.M. Shneidman, G.G. Adamian, N.V. Antonenko, R.V. Jolos, W. Scheid (2003) Phys. Rev. C 67014313.

[9] T.M. Shneidman, G.G. Adamian, N.V. Antonenko, R.V. Jolos, W. Scheid (2011) Eur. Phys. J. A 4734. 


\section{E.V. Mardyban, T.M. Shneidmanr, N.V. Antonenko, G.G. Adamian}

[10] T.M. Shneidman, G.G. Adamian, N.V. Antonenko, R.V. Jolos, S.-G. Zhou (2015) Phys. Rev. C 92034302.

[11] M. Spieker et al. (2018) Phys. Rev. C 97064319.

[12] M. von Tresckow et al. (2021) Phys. Lett. B 821136624.

[13] L.P. Gaffney et al. (2013) Nature (London) 497199.

[14] P.A. Butler et al. (2020) Phys. Rev. Lett. 124042503.

[15] G.G. Adamian, N.V. Antonenko, R.V. Jolos, S.P. Ivanova, O. Melnikova (1996) Int. J. Mod. Phys. E 5191.

[16] G.G. Adamian, N.V. Antonenko, R.V. Jolos (1995) Nucl. Phys. A 584205.

[17] G. Audi, O. Bersillon, J. Blachot, A.H. Wapstra (2003) Nucl. Phys. A 7293.

[18] E.V. Mardyban, T.M. Shneidman, E.A. Kolganova, R.V. Jolos, S.-G. Zhou (2020) Chin. Phys. C $\mathbf{4 2} 124104$.

[19] R.V. Jolos, P. von Brentano, F. Doüau (1993) J. Phys. G 19151.

[20] http://www.nndc.bnl.gov/nndc/ensdf.

[21] H.J. Wollersheim et al. (1993) Nucl. Phys. A 556, 261

[22] A. Bohr, B.R. Mottelson (1975) "Nuclear Structure” Vol. II (Benjamin, New York).

[23] E.T.Gregor et al. (2019) J. Phys. G 46075101. 